\title{
Comorbilidades asociadas a mortalidad materna por COVID-19
} en México

\section{Guadalupe López-Rodríguez *, Marcos Galván y Oscar Galván Valencia²}

${ }^{1}$ Área Académica de Nutrición, Instituto de Ciencias de la Salud, Universidad Autónoma del Estado de Hidalgo, Pachuca de Soto, Hgo.; ${ }^{2}$ Instituto de Nutrición, Universidad de la Sierra Sur, Miahuatlán de Porfirio Díaz, Oax. México

\section{Resumen}

Introducción: En México existe una sindemia en las mujeres gestantes, donde coexiste la epidemia de obesidad y enfermedades crónicas con la de enfermedad por coronavirus 2019 (COVID-19), lo que se ha asociado con un mayor riesgo de mortalidad. Objetivo: Evaluar la asociación de las comorbilidades en la mortalidad materna por COVID-19 en México. Material y métodos: Se utilizaron las bases de datos de COVID-19 del SISVER y los reportes de vigilancia epidemiológica de muertes maternas. Se utilizaron modelos de regresión logística multivariante para evaluar la asociación de las comorbilidades durante la gestación con mortalidad materna por COVID-19. Resultados: Se evaluaron 29,416 mujeres embarazadas, el 39\% fueron positivas para coronavirus 2 del síndrome respiratorio agudo grave, el riesgo de mortalidad materna fue 3.24 veces mayor $(p<0.01)$ para las mujeres positivas en comparación con las negativas. La COVID-19 es la primera causa de muerte materna desde julio del 2020 y explica más del 50\% del total de muertes en el 2021. La enfermedad renal crónica (razón de momios [RM]: 4.11; $p$ < 0.01) y la diabetes (RM: 2.53; $p<0.01$ ) fueron las dos principales comorbilidades asociadas positivamente a la mortalidad materna por COVID-19. Conclusión: Las comorbilidades durante el embarazo posiblemente asociadas con un incremento de la respuesta inflamatoria y alteración de la respuesta inmunitaria incrementan el riesgo de muerte materna por COVID-19 en mujeres gestantes mexicanas.

PALABRAS CLAVE: Comorbilidad. COVID-19. Mortalidad materna. SARS-CoV-2.

\section{Comorbidities associated with maternal mortality from COVID-19 in Mexico}

\section{Abstract}

Introduction: In Mexico, there is a syndemic in pregnant women, where the epidemic of obesity and chronic diseases coexists with that of coronavirus disease 2019 (COVID-19), which has been associated with a higher risk of maternal mortality. Objective: To evaluate the association of comorbidities during pregnancy with maternal mortality from COVID-19 in Mexico. Material and methods: SISVER COVID-19 databases and epidemiological surveillance reports on maternal mortality were used. Multivariate logistic regression models were used to evaluate the association of comorbidities with maternal deaths from COVID-19. Results: A total of 29,416 pregnant women were evaluated, out of which $39 \%$ were positive for severe acute respiratory syndrome coronavirus 2 (SARS-CoV-2); the risk of maternal mortality was 3.24 times higher $(p<0.01)$ for positive in comparison with negative women. COVID-19 is the leading cause of maternal death since July 2020 and explains more than $50 \%$ of total deaths in 2021. Chronic kidney disease (odds ratio [OR]: 4.11; $p<0.01$ ) and diabetes (OR: 2.53; $p<0.01)$ were the two main comorbidities that were positively associated with maternal death from COVID-19. Conclusion: Comorbidities during pregnancy that are possibly associated with an increase in the inflammatory response and an alteration of the immune response increase the risk of maternal death from COVID-19 in Mexican pregnant women.

KEY WORDS: Comorbidity. COVID-19. Maternal mortality. SARS-CoV-2.

Correspondencia:

*Guadalupe López-Rodríguez

E-mail: glopez.dra@gmail.com; glopez@uaeh.edu.mx

DOI: 10.24875/GMM.21000221
Gac Med Mex. 2021;157:618-622

Disponible en PubMed

www.gacetamedicademexico.com

CC BY-NC-ND (http://creativecommons.org/licenses/by-nc-nd/4.0/). 


\section{Introducción}

Desde diciembre de 2019 aparecieron múltiples casos de neumonía de etiología desconocida en Wuhan, provincia de Hubei, China, y posteriormente se identificó un nuevo coronavirus que se denominó coronavirus 2 del síndrome respiratorio agudo grave (SARS-CoV-2) ${ }^{1}$. La obesidad es una de las principales patologías asociada con evolución grave de la enfermedad por coronavirus 2019 (COVID-19). A nivel mundial se estima que más de dos billones de personas tienen sobrepeso $u$ obesidad ${ }^{2}$, lo que les predispone al desarrollo de otras patologías crónicas ${ }^{3}$. La encuesta nacional de salud y nutrición (ENSANUT) en el 2018 reportó que en México el $75.2 \%$ de los adultos de 20 años y más tienen sobrepeso y obesidad, de los cuales el $76.8 \%$ son mujeres ${ }^{4}$. La obesidad en mujeres se relaciona con un mayor riesgo de enfermedades cardiovasculares, diabetes, hipertensión, trastornos ginecológicos ${ }^{5}$ y enfermedades infecciosas ${ }^{6}$.

Existe una mayor probabilidad de que las mujeres gestantes sufran complicaciones y enfermedades por infecciones, incluida la del SARS-CoV-2. Debido a esto, las gestantes son un grupo vulnerable durante cualquier emergencia sanitaria ${ }^{7}$, en vista de que durante el embarazo existen cambios fisiológicos caracterizados por una adaptación en la respuesta inmunitaria ${ }^{8}$, lo que incrementa el riesgo y la gravedad de las infecciones. Además, durante el embarazo existe una acumulación fisiológica de tejido adiposo, que se asocia con un aumento en la respuesta inflamatoria ${ }^{9}$, que es mayor si existe obesidad pregestacional ${ }^{10}$.

En las mujeres gestantes el SARS-CoV-2 puede causar complicaciones como abortos, restricción del crecimiento fetal y parto prematuro, lo que incrementa el riesgo de sufrimiento fetal y muerte materna ${ }^{11}$. En México no existen reportes de las comorbilidades asociadas con las muertes maternas, por lo que el objetivo de este estudio fue evaluar la asociación entre las comorbilidades durante la gestación sobre el riesgo de mortalidad en mujeres gestantes con diagnóstico de COVID-19.

\section{Metodología}

En este estudio se utilizó la base de datos abiertos del Sistema Nacional de Vigilancia Epidemiológica de Enfermedades Respiratorias Virales (SISVER) para casos de COVID-19 en México disponibles al 2 de febrero del $2021^{12}$ y los Informes Semanales para la Vigilancia Epidemiológica de Muertes Maternas del $2020^{13}$ y $2021^{14}$. En la base del SISVER se seleccionaron todas las mujeres gestantes con análisis de laboratorio para detectar la presencia de SARS-CoV-2 con una prueba molecular, mediante el método de reacción en cadena de la polimerasa $(\mathrm{PCR})^{15}$.

Se realizaron análisis descriptivos de las principales características de interés. Las variables categóricas se describieron como porcentajes. Se realizaron pruebas de regresión logística multivariante para la asociación de la mortalidad materna con los diagnósticos de diabetes, hipertensión (HTA), enfermedad pulmonar obstructiva crónica (EPOC), asma, inmunológica, cardiovascular, enfermedad renal crónica (ERC) y obesidad registrados en la base de datos del SISVER. Además se realizaron modelos de regresión logística bivariados para asociar la mortalidad materna con las pruebas positivas para SARS-CoV-2; se incluyó al análisis la edad materna y las comorbilidades para estimar la razón de momios (RM) y los intervalos de confianza al 95\% (IC 95\%). El valor de $p<0.05$ se consideró estadísticamente significativo. El análisis de los datos se realizó utilizando el paquete estadístico SPSS versión 21 (SPSS Corp., Chicago, IL, EE.UU.).

\section{Resultados}

Se evaluaron 11,488 registros de mujeres positivas para SARS-CoV-2 y 17,928 con resultado negativo, el porcentaje de positividad fue del 39\%. La RM de mortalidad de una mujer embarazada con prueba positiva para SARS-CoV-2 fue de 3.24 (IC 95\%: 2.3-4.5) en comparación con las gestantes con resultados negativos para la prueba. En la figura 1 se muestra el número acumulado de muertes maternas por COVID-19 en México, al 8 de marzo del 2021 son 304 muertes, de las cuales 202 se registraron en 2020 y 102 en el 2021 . En el 2020 los dos primeros casos se registraron en la semana epidemiológica 15 y representaban el 1.3\% del total de causas de muerte; en la semana 28 la COVID-19 se ubicó como la primera causa de muerte (17.5\% del total). De la semana epidemiológica 33 a la 53 , el porcentaje de muertes por COVID-19 se mantuvo alrededor del 22\%. Desde inicio del 2021 se presentó un incremento súbito en el número de muertes maternas confirmadas por COVID-19 (Fig. 2), representando en la semana epidemiológica 1 el $61.3 \%$ de las 
Tabla 1. Razón de momios de la mortalidad materna por patología en mujeres gestantes mexicanas con diagnóstico de enfermedad por coronavirus 2019 (COVID-19)*

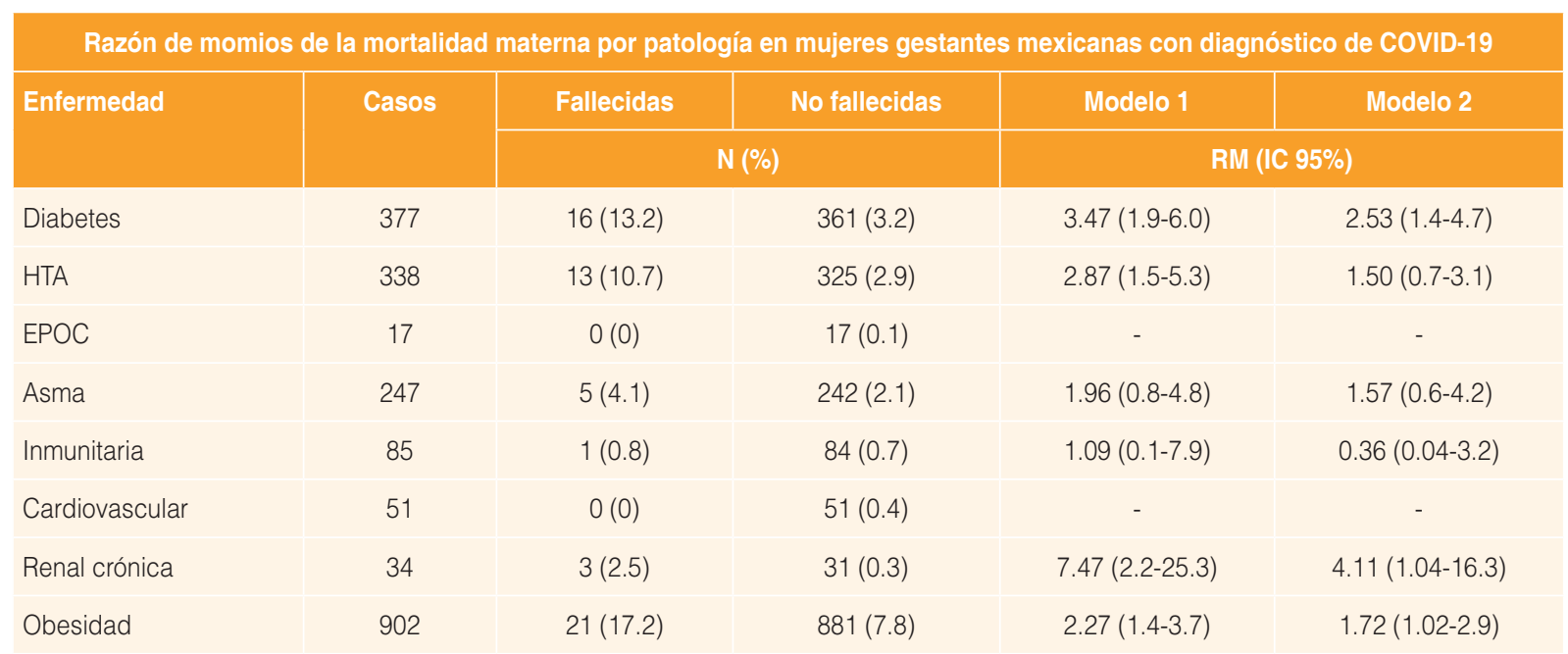

RM: razón de momios; IC 95\%: intervalo de confianza al 95\%; HTA: hipertensión; EPOC: enfermedad pulmonar obstructiva crónica

*Tabla elaborada con registros del informe del 2 de febrero del $2021 .{ }^{12}$ El total de mujeres fallecidas registradas fueron 122 y de no fallecidas de 11,346 . En el modelo 1 se ajustó por edad materna y en el modelo 2 por edad materna y todas las comorbilidades del modelo 1.

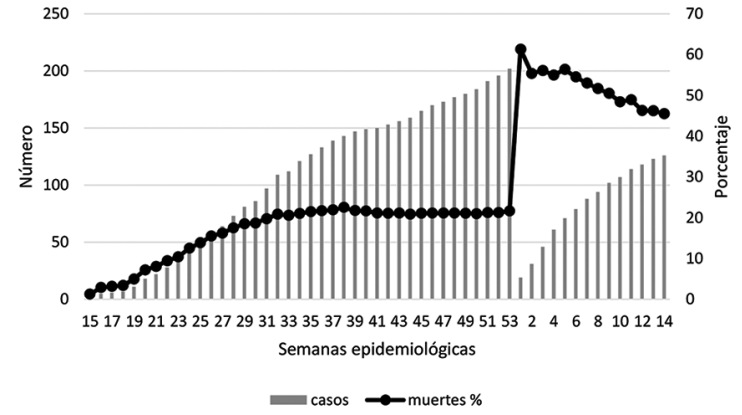

Figura 1. Muertes maternas acumuladas por enfermedad por coronavirus 2019 (COVID-19) en México, 2020 y 2021. Los datos de las barras representan el número de casos acumulados para COVID-19. La línea indica el porcentaje de muerte materna por COVID-19 en relación con el total de muertes registradas por semana epidemiológica. Informe al 12 de abril del 2021.

muertes maternas y en la semana epidemiológica 14 está cerca del $50 \%$ (Fig. 1).

En la tabla 1 se presentan las comorbilidades asociadas a la mortalidad materna. En el modelo 1 se observó que las enfermedades que se asociaron con un mayor riesgo de mortalidad por COVID-19 fueron la ERC (RM: 7.47; IC 95\%: 2.2-25.3), seguida por la diabetes (RM: 3.47; IC 95\%: 1.9-6.0) y la HTA (RM: 2.87; IC 95\%: 1.5-5.3) con un riesgo de 7.47, 3.47 y 2.87, respectivamente (modelo $1, p<0.05$ ). Sin embargo, en el modelo 2 solo se mantiene la asociación para diabetes (RM: 2.53; IC 95\%: 1.4-4.7), obesidad (RM: 1.72; IC 95\%: 1.02-2.9) y ERC (RM: 4.11;

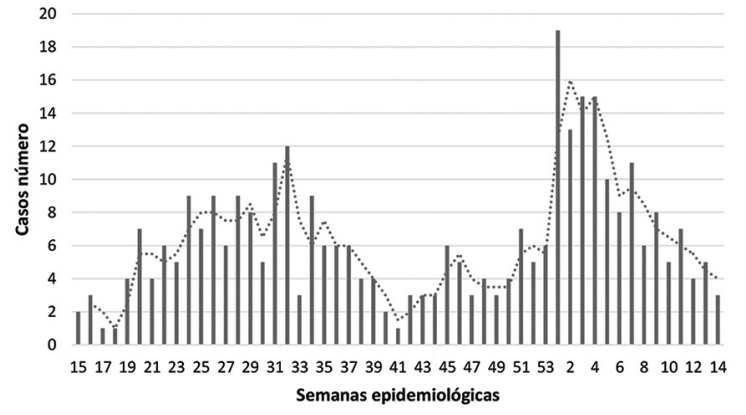

Figura 2. Casos de enfermedad por coronavirus 2019 (COVID-19) en México, 2020 y 2021. Los datos de las barras representan el número de casos confirmados para COVID-19. Elaborado con datos de los informes de las semanas epidemiológicas del 2020 (desde la semana 15) y hasta la semana epidemiológica 14 del año 2021.

IC 95\%: 1.04-16.3). La EPOC y la enfermedad cardiovascular no se asociaron con el riesgo de mortalidad en mujeres embarazadas con diagnóstico positivo para SARS-CoV-2.

\section{Discusión}

En el presente estudio se observó una asociación positiva entre la presencia de enfermedades crónicas durante el embarazo con una mayor posibilidad de muerte por COVID-19. En las mujeres gestantes existe controversia respecto al riesgo de complicaciones y muerte por COVID-19, los primeros 
estudios en México indicaban que la gestación no implicaba un mayor riesgo al compararse con mujeres no gestantes ${ }^{16}$; sin embargo, los últimos reportes en México ${ }^{17,18}$ y otros países muestran lo contrario $^{19}$. En México la COVID-19 es una condición de riesgo de muerte, en este estudio se identificó en las mujeres gestantes una RM de 3.24 $(p<0.001)$; la COVID-19 se caracteriza por disminuir los linfocitos e incrementar las citocinas proinflamatorias, lo que también se observa durante el embarazo, además de que la placenta tiene receptores para la enzima conversiva de la angiotensina (ECA) $2^{20}$, lo que en conjunto potenciaría el riesgo de complicaciones y muerte asociado a la infección por el SARS-CoV-2.

La mortalidad por la infección también se asocia con las comorbilidades que presenta la mujer gestante, siendo la enfermedad renal la que registró el riesgo más alto (RM: 4.11; IC 95\%: 1.3-23.6); en un estudio previo se observó una RM: 2.25 (IC 95\%: 1.04-16.3) en mujeres mexicanas con COVID-19 ${ }^{21}$ y de 1.99 (IC 95\%: 1.9-2.1) en población general. Sin embargo, en el presente estudio se observó que el riesgo es mucho mayor cuando las mujeres están en gestación. Esto posiblemente se asocia con la inmunosupresión que se ha identificado en los pacientes renales $\operatorname{crónicos}^{22}$. Además de que muchos de estos pacientes son tratados con fármacos inhibidores de la ECA1 y bloqueadores del receptor de angiotensina, debido al incremento en la actividad del sistema renina-angiotensina-aldosterona, lo cual incrementa la expresión de ECA2 en hígado y corazón ${ }^{23}$.

En México la obesidad es la principal comorbilidad asociada con el riesgo de manifestaciones graves ${ }^{24} y$ de muerte ${ }^{25}$ por COVID-19, lo cual no se confirma en las mujeres gestantes evaluadas, en quienes la diabetes y la ERC son el principal riesgo asociado a mortalidad. Este resultado tiene limitaciones, debido a que la evaluación y diagnóstico de obesidad durante la gestación debe realizarse por un experto y en este estudio fue por autorreporte. Sin embargo, estos resultados resaltan la importancia de estas enfermedades dentro de la carga global de la enfermedad en la población en México.

\section{Conclusiones}

La COVID-19 es la principal causa de mortalidad materna en México desde la segunda semana de julio del 2020 y a un año de inicio de la pandemia continúa en el mismo sitio, explicando el $27 \%$ de las muertes maternas. Al mes de marzo del 2021, los contagios por COVID-19 en mujeres embarazadas, correspondientes a la segunda ola, están en descenso.

La ERC, la diabetes y la obesidad tienen un efecto importante sobre la mortalidad materna asociada a COVID-19 en México. Estas patologías se asocian con una mayor respuesta inflamatoria y alteración de la respuesta inmunitaria, lo que asociado con la gestación potenciaría los riesgos de formas graves y muerte ante la infección por SARS-CoV-2.

\section{Financiamiento}

La presente investigación no ha recibido ayudas específicas provenientes de agencias del sector público, sector comercial o privadas.

\section{Conflicto de intereses}

Los otros autores declaran no tener ningún conflicto de interés.

\section{Responsabilidades éticas}

Protección de personas y animales. Los autores declaran que para esta investigación no se han realizado experimentos en seres humanos ni en animales.

Confidencialidad de los datos. Los autores declaran que en este artículo no aparecen datos de pacientes.

Derecho a la privacidad y consentimiento informado. Los autores declaran que en este artículo no aparecen datos de pacientes.

\section{Bibliografía}

1. Zhu N, Zhang D, Wang W, Li X, Yang B, Song J, et al. A novel coronavirus from patients with pneumonia in China, 2019. N Engl J Med. 2020;382(8):727-33.

2. Caballero B. Humans against Obesity: Who will win? Adv Nutr. 2019;10(Suppl 1):S4-S9.

3. Saltiel AR, Olefsky J. Inflammatory mechanisms linking obesity and metabolic disease. J Clin Invest. 2017;127(1):1-4.

4. Encuesta Nacional de Salud y Nutrición 2018-19. Resultados nacionales [Internet]. México: Centro de Investigación en Evaluación y Encuestas. Disponible en: https://ensanut.insp.mx/encuestas/ensanut2018/informes.php

5. Tauqeer Z, Gomez G, Stanford FC. Obesity in women: Insights for the clinician. J Womens Health (Larchmt). 2018;27(4):444-57.

6. Fezeu L, Julia C, Henegar A, Bitu J, Hu FB, Grobbee DE, et al. Obesity is associated with higher risk of intensive care unit admission and death in influenza A (H1N1) patients: a systematic review and meta-analysis. Obes Rev. 2011;12(8):653-59.

7. López-Rodríguez G, Alvarez J, Galván M, Montiel-Hernández R. Embarazo y COVID-19, un enfoque preventivo para la salud nutricional. Educación y Salud. Boletín Científico Instituto de Ciencias de la Salud Universidad Autónoma del Estado de Hidalgo. 2020;9(17):45-53.

8. Soma-Pillay P, Nelson-Piercy C, Tolppanen H, Mebazaa A. Physiological changes in pregnancy. Cardiovasc J Afr. 2016;27(2):89-94.

9. Ovalle A, Martínez MA, Fuentes A, Marques X, Vargas F, Vergara P, et al. Obesity, a risk factor for ascending bacterial infection during pregnancy. Rev Med Chile. 2016;144(4):476-82. 
10. Zacarias MF, Collado MC, Gomez-Gallego C, Flinck H, Aittoniemi J, Isolauri E, et al. Pregestational overweight and obesity are associated with differences in gut microbiota composition and systemic inflammation in the third trimester. PLoS One. 2018;13(7):e0200305.

11. Panahi L, Amiri M, Pouy S. Risks of novel coronavirus disease (COVID-19) in pregnancy; a narrative review. Arch Acad Emerg Med. 2020;8(1):34

12. Secretaría de Salud. Bases de datos COVID 19 en México 2021 [Internet]. Gobierno de México, Secretaría de Salud. Disponible en: https:// www.datos.gob.mx/busca/dataset/informacion-referente-a-casos-covid-19-en-mexico

13. Secretaría de Salud. Informes Semanales para la Vigilancia Epidemiológica de Muertes Maternas 2020 [Internet]. Gobierno de México, Secretaría de Salud; 2020. Disponible en: https://www.gob.mx/salud/documentos/informes-semanales-para-la-vigilancia-epidemiologica-de-muertes-maternas-2020

14. Secretaría de Salud. Informes Semanales para la Vigilancia Epidemiológica de Muertes Maternas 2021 [Internet]. Gobierno de México, Secretaría de Salud; 2021. Disponible en: https://www.gob.mx/salud/documentos/informes-semanales-para-la-vigilancia-epidemiologica-de-muertes-maternas-2021

15. Tang YW, Schmitz JE, Persing DH, Stratton CW. Laboratory diagnosis of COVID-19: Current issues and challenges. J Clin Microbiol. 2020;58(6):e00512-20.

16. Rios-Silva M, Murillo-Zamora E, Mendoza-Cano O, Trujillo X, Huerta M. COVID-19 mortality among pregnant women in Mexico: A retrospective cohort study. J Glob Health. 2020;10(2):020512.

17. Martinez-Portilla RJ, Sotiriadis A, Chatzakis C, Torres-Torres J, Espino YSS, Sandoval-Mandujano $\mathrm{K}$, et al. Pregnant women with SARS-CoV-2 infection are at higher risk of death and pneumonia: propensity score matched analysis of a nationwide prospective cohort (COV19Mx). Ultrasound Obstet Gynecol. 2021;57(2):224-31.
18. Martinez-Portilla RJ, Smith ER, He S, Torres-Torres J, Espino YSS, Solis-Paredes JM, et al. Young pregnant women are also at an increased risk of mortality and severe illness due to coronavirus disease 2019: analysis of the Mexican National Surveillance Program. Am J Obstet Gynecol. 2021;224(4):404-7.

19. Subbaraman N. Pregnancy and COVID: what the data say. Nature. 2021:591(7849):193-5.

20. Phoswa WN, Khaliq OP. Is pregnancy a risk factor of COVID-19? Eur J Obstet Gynecol Reprod Biol. 2020;252:605-9.

21. Hernandez-Garduno E. Obesity is the comorbidity more strongly associated for Covid-19 in Mexico. A case-control study. Obes Res Clin Pract. 2020;14(4):375-9.

22. Girndt M, Sester M, Sester U, Kaul H, Kohler H. Molecular aspects of T- and B-cell function in uremia. Kidney Int Suppl. 2001;78:S206-11.

23. Jessup JA, Gallagher PE, Averill DB, Brosnihan KB, Tallant EA, Chappell MC, et al. Effect of angiotensin II blockade on a new congenic model of hypertension derived from transgenic Ren-2 rats. Am J Physiol Heart Circ Physiol. 2006;291(5):H2166-72.

24. Denova-Gutierrez E, Lopez-Gatell H, Alomia-Zegarra JL, Lopez-Ridaura R, Zaragoza-Jimenez CA, Dyer-Leal DD, et al. The association of obesity, type 2 diabetes, and hypertension with severe coronavirus disease 2019 on admission among Mexican patients. Obesity (Silver Spring). 2020;28(10):1826-32.

25. Bello-Chavolla OY, Bahena-Lopez JP, Antonio-Villa NE, Vargas-Vazquez A, Gonzalez-Diaz A, Marquez-Salinas A, et al. Predicting mortality due to SARS-CoV-2: A mechanistic score relating obesity and diabetes to COVID-19 outcomes in Mexico. J Clin Endocrinol Metab. 2020;105(8):dgaa346 